\title{
Biochemical and structural studies of plant circadian clock proteins
}

\author{
Reena Saini ${ }^{1,3}$, Kamil SzPotkowski ${ }^{1}$, Maciej KozaK ${ }^{2}$, Mariusz Jaskolski ${ }^{1,2 *}$, Seth J. DaVis ${ }^{3}$ \\ ${ }^{1}$ Institute of Bioorganic Chemistry, Polish Academy of Sciences, Poznań, Poland \\ ${ }^{2}$ Faculty of Chemistry, Adam Mickiewicz University, Poznań, Poland \\ ${ }^{3}$ Max-Planck-Institut für Pflanzenzüchtungsforschung, Cologne, Germany \\ *Corresponding author: mariuszj@amu.edu.pl
}

Circadian clocks are biochemical oscillatory mechanisms that co-ordinate with a $24 \mathrm{~h}$ period predicting the daily diurnal changes in the environment to regulate various metabolic, developmental and physiological processes in the organisms. The plant circadian clock represents a network of interconnected multiple feedback loops at the core of which lie two Myb-like transcription factors, Circadian Clock Associated 1 (CCA1) and Late Elongated Hypocotyl (LHY), that express in the morning, and an evening expressing Timing of $\mathrm{CAB}$ Expression 1 (TOC1). Other components important for the clock are Early Flowering 3 and 4 (ELF3 and ELF4) and Lux Arrhythmo (LUX) that accumulate in the evening (Harmer, 2009). Previous studies have shown these to be part of an evening complex that is important for the regulation of the core oscillator (Nusinow et al., 2011; Herrero et al., 2012). Loss-of-function mutation studies of these components demonstrate arrhythmia under constant conditions of light (LL) and in darkness (DD) (Kolmos et al., 2009; Thines and Harmon, 2010). Recently, it has been shown (both in vivo and in vitro) that ELF3 and ELF4 interact physically with each other to perform their regulatory function (Herrero et al., 2012).

Here, using various in silico and experimental approaches, we have tried to understand the structural aspects of these two evening expressing plant clock proteins (ELF3 and 4). Due to the instability of the ELF4 and ELF3 proteins, crystallization and Small Angle X-ray Scattering (SAXS) measurements were carried out for recombinant variants tagged with fusion (MBP or NusA) proteins.

\section{Crystallization of ELF4 and ELF3}

Commercially available crystallization screens were used with purified recombinant fusion proteins. Crystals

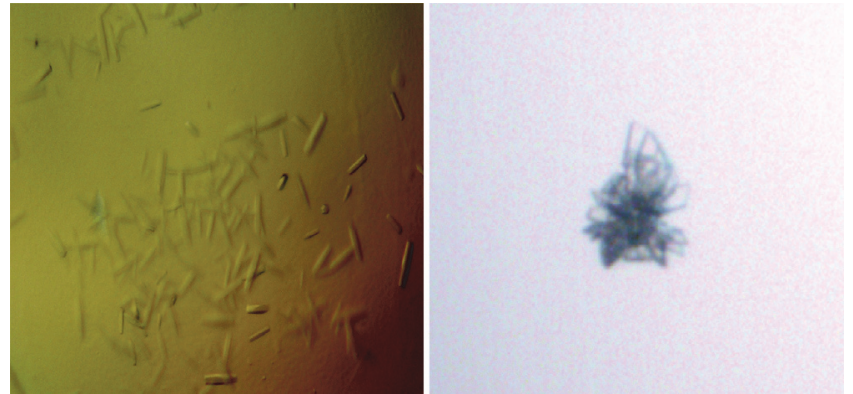

Fig. 1. Preliminary crystals obtained for His-NusA-ELF4

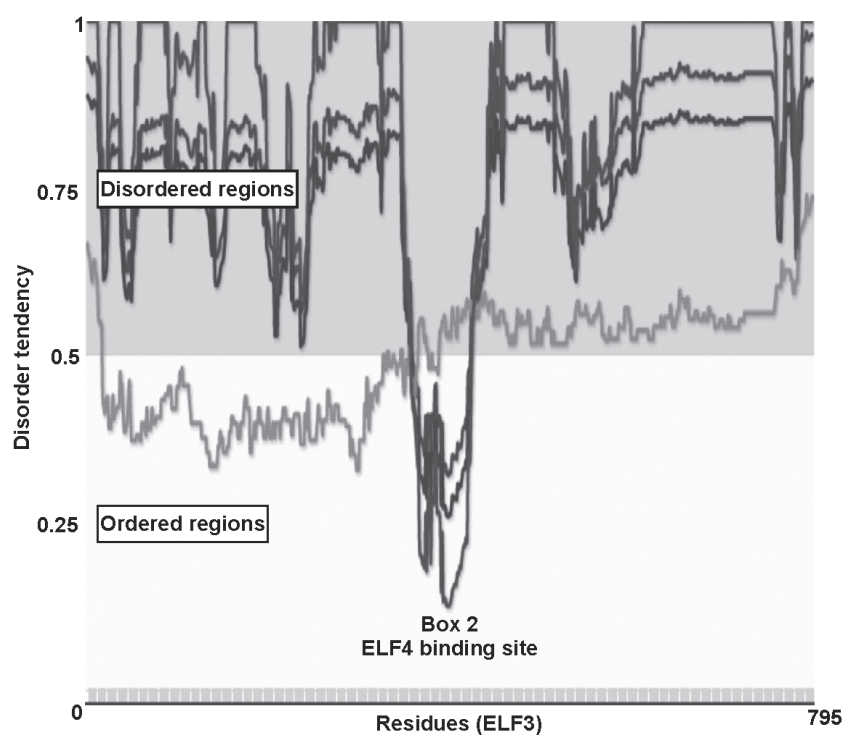

Fig. 2. Predicted regions of order/disorder in ELF3 (predictions calculated using Metadisorder: Kozlowski et al., 2012)

were observed for NusA tagged ELF4 in two conditions. However, the crystals could not be obtained again. The crystals observed in the screens dissolved after a few days; hence, data could not be collected (Fig. 1). No crystals were observed for ELF3 recombinant protein variants. 


\section{In silico analysis of ELF3}

We analyzed the ELF3 protein sequences from different species and observed four small regions of high conservation throughout the entire sequence. The highly conserved Box 2 corresponds to the ELF4 binding site. On the basis of results obtained from $a b$ initio computational modeling, we predict that Box 2 might also exhibit structural conservation. Overall, ELF3 showed large regions of sequence consistent with intrinsic disorder, except in the Box 2 region (Fig. 2). We hypothesize that ELF3 belongs to the class of intrinsically unstructured proteins (IUP) that are involved in multiple functions via forming transient complexes with their various interacting partners.

\section{SAXS analysis of ELF3 and 4}

Scattering data for the recombinant fusion proteins were collected at BioSAXS beam line BM29 of the storage ring at the European Synchrotron Radiation Facility (Grenoble, France). A Kratky plot for the analyzed recombinant proteins represents the scattering pattern as $s^{2} I(s)$ versus $s$ (Fig. 3).

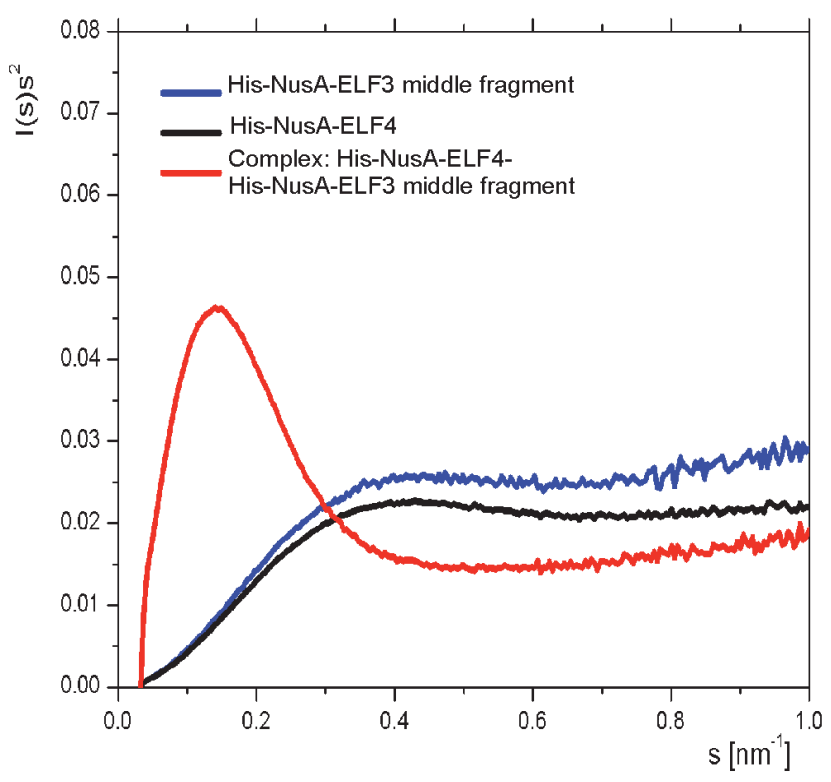

Fig. 3. Kratky plot for recombinant fusion proteins: ELF4, ELF3 middle fragment and the complex between the ELF4 and ELF3 middle fragment (1:1)
In Kratky plot, globular proteins show an intense maximum due to a highly ordered inner structure, whereas unfolded proteins exhibit a plateau (Putnam et al., 2007). A Kratky plot for recombinant ELF4 and ELF3 tagged variants showed a plateau, suggesting that the proteins might be unstructured and/or highly dynamic. For the complex His-NusA-ELF4 : His-NusA-ELF3 (middle fragment) (1:1 ratio) a maximum is visible indicating an increase in the ordered structure, suggesting that proteinprotein interaction contributes to the stability of the proteins in the complex, which is a pre-requisite for their function in the regulation of the core oscillator.

\section{References}

Harmer S.L. (2009) Annu. Rev. Plant Biol. 60: 357-377.

Kolmos E., Nowak M., Werner M., Fischer K., Schwarz G., Mathews S., Schoof H., Nagy F., Bujnicki J.M., Davis S.J. (2009) Front. Life Sci. (formerly HFSP J.) 3: 350-366.

Thines B., Harmon F.G. (2010) Proc. Natl Acad. Sci. USA 107(7): 3257-3262.

Nusinow D.A., Helfer A., Hamilton E.E., King J.J., Imaizumi T., Schultz T.F., Farré E.A., Kay S.A. (2011) Nature 475: 398-402.

Herrero E., Kolmos E., Bujdoso N., Yuan Y., Wang M., Berns M.C., Coupland G., Saini R., Jaskolski M., Webb A., Gonçalves J., Davis S.J. (2012) Plant Cell 24(2): 428-443.

Kozlowski L.P., Bujnicki J.M. (2012) BMC Bioinformatics 24 13(1): 111.

Putnam C.D., Hammel M., Hura G.L., and Tainer J.A. (2007) Q. Rev. Biophys. 40(3): 191-285. 